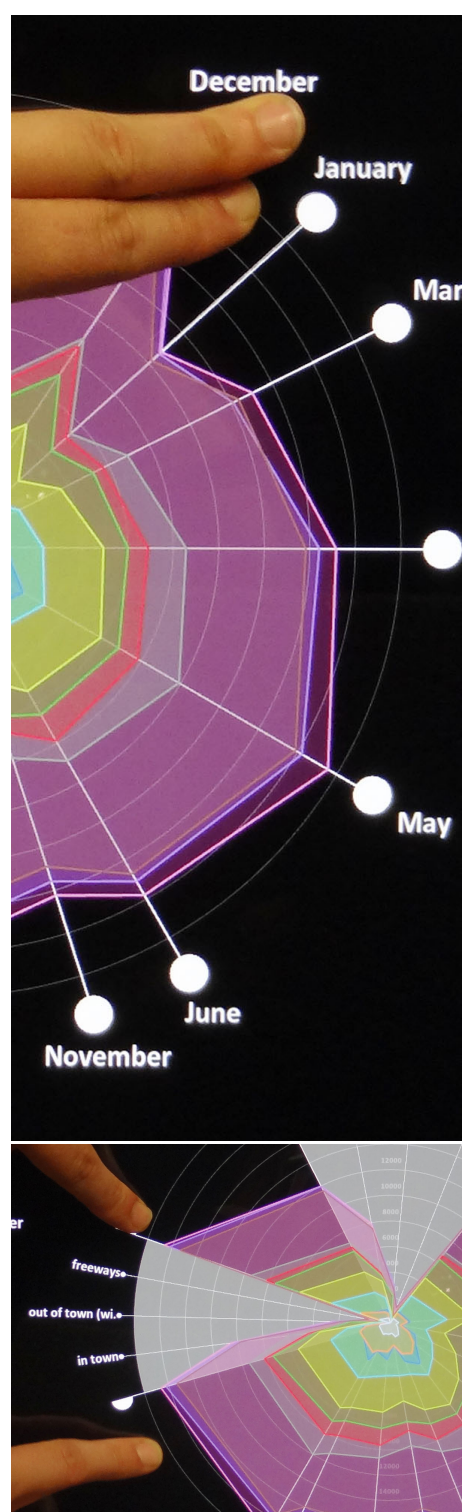

Figure 1: A multi-touch interaction concept for star plots: rearranging axes ( $\uparrow$ top), splitting up an axis to explore a second information layer $(\downarrow$ bottom)

\section{Towards a Fluid Interaction Concept Bringing Star Plots to Interactive Displays}

\author{
Ricardo Langner \\ Interactive Media Lab \\ Technische Universität Dresden Technische Universität Dresden \\ Dresden, Germany \\ langner@acm.org \\ Ulrike Kister \\ Interactive Media Lab \\ Technische Universität Dresden \\ Dresden, Germany \\ ukister@acm.org \\ Tom Horak \\ Dresden, Germany \\ horakt@acm.org \\ Raimund Dachselt \\ Interactive Media Lab \\ Dresden, Germany \\ dachselt@acm.org
}

Christin Engel

Interactive Media Lab

Technische Universität Dresden

Dresden, Germany

christin.engel@mailbox.tu-

dresden.de

Permission to make digital or hard copies of part or all of this work for personal or classroom use is granted without fee provided that copies are not made or distributed for profit or commercial advantage and that copies bear this notice and the full citation on the first page. Copyrights for third-party components of this work must be honored. For all other uses, contact the Owner/Author.

Copyright is held by the owner/author(

ITS '15, November 15-18, 2015, Funchal, Portugal

ACM 978-1-4503-3899-8/15/11. http://dx.doi.org/10.1145/2817721.2823505

\section{Abstract}

In this paper, we present several concepts towards fluid multi-touch interactions for an interactive star plot visualization. The goal of our research is to improve the usability of visualizations by developing new and natural interaction techniques as well as designing and applying new visualization approaches. To achieve this, we systematically create consistent touch interactions for various tasks typical to information visualizations. Furthermore, we propose a new approach to integrate additional levels of information into a star plot by splitting up axes. Finally, we have successfully implemented many of our concepts in a first prototype, allowing the validation of the usefulness and usability.

\section{Author Keywords}

Information visualization; Star plots; Multi-touch; Fluid interaction.

\section{ACM Classification Keywords}

H.5.2. [Information Interfaces and Presentation (e.g. $\mathrm{HCl}$ )] User Interfaces

\section{Introduction \& Background}

Combining the two research fields of information visualization and natural user interfaces is an upward trend [6]. On the one hand, the use of touch-enabled devices such as 
mobile phones, tablets, or interactive tabletops is promising $[1,3,8]$, since these devices have become ubiquitous in many places. Direct touch interaction can make the exploration and analysis of data more attractive to users and lower the bar for inexperienced users. On the other hand, dealing with multivariate (i.e., hypervariate) data is an important visualization challenge. Established approaches are coordinate plots such as parallel coordinate plots or star plots.

This is not the first paper addressing multi-touch interfaces for information visualization. Existing work, however, investigated different visualization techniques; a consistent set of natural interactions for star plots has not been developed. Baur et al. developed TouchWave [1] to explore touch interaction for stacked graphs. Drucker et al. [3] investigate interaction bar charts and compare a traditional desktop interface with a new touch interfaces for touch-enabled mobile devices. Sadana and Stasko [8] created an interactive scatterplot visualization for tablet computer and started exploring the design space of multi-touch interactions for visualizations. Rzeszotarski and Kittur [7] use physics-based affordances for data exploration and also compared a touch prototype for tablets with a traditional spreadsheet tool.

In our research, we investigate multi-touch interaction concepts for star plots. Star plots support the understanding of multivariate data objects as visual shapes with diverse properties (Figure 1). These shapes can be used for comparison of data using their glyph characteristics [9]. It is also possible to compare attribute values to the extremes and the distribution of values along the axis [5].

We contribute a consistent touch interface for star plots designed for interactive displays. We investigated specific mappings of touch interactions to visualization tasks, the interplay of different visualization components, and

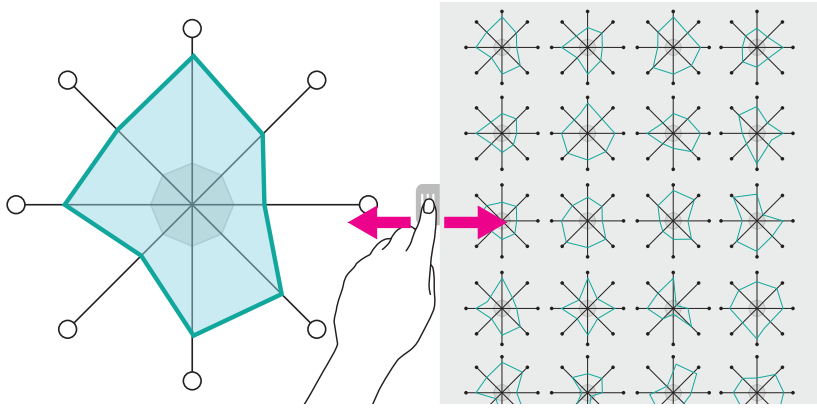

Figure 2: Interface Composition: highly interactive detail view (left), matrix view (right) showing small glyph-like star plots.

the extension of the functionality of star plots. Among others, we designed new interactions for (i) the dynamic rearrangement of one or multiple axes and (ii) the exploration of (semi-)structured data through splitting up axes.

In the following, we will first describe our process and general principles for the design of the multi-touch interface for star plots. We will thereby propose concepts extending the existing star plot technique to visualize (semi-)structured data. Finally, we briefly present our prototype implementation and present first insights.

\section{A Multi-Touch Interface for Star Plots}

In the following, we propose specific multi-touch concepts for star plots. We systematically designed solutions for various tasks typical of information visualization. Therefore, our solutions are classified following the categories of interactions identified by Yi et al. [10]: Select, Explore, Reconfigure, Encode, Abstract/Elaborate, Filter, and Connect.

We specifically focus on designing natural interactions and apply phrasing [2] for a seamless interaction style. According to the guidelines of fluid interaction [4] as well as results 

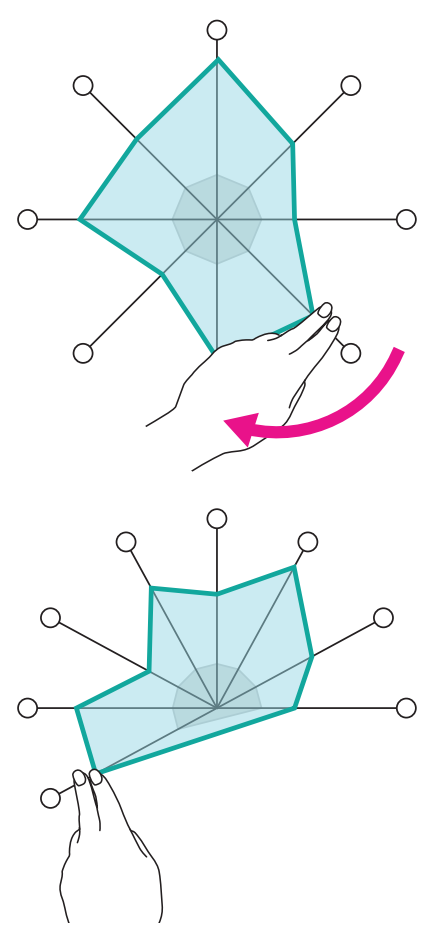

Figure 3: Axes arrangement: axes are pushed in the direction of the movement.

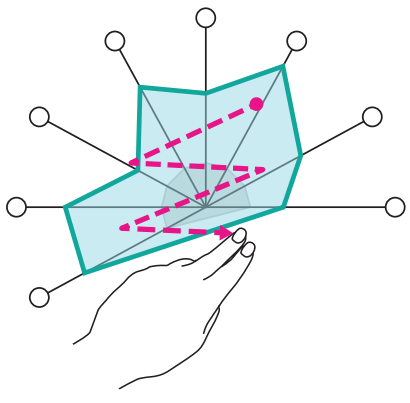

Figure 4: Wipe gesture to reset the distribution of axes. from Drucker et al. [3], we focus on making the data and visual elements themselves interactive. We use local on-site extensions that allow direct manipulation of the visualization (e.g., interactive axes, data objects, intersections), rather than menus or separated visualization panes.

\section{Interface Composition \& General Principles}

Typical of star plot visualizations, our interface consists of two major components (Figure 2): a detail view and a matrix view. The highly interactive detail view allows users to read and compare attribute values by showing one or more selected data objects in a single, large coordinate view. The matrix view is located directly beside the detail view. It shows data objects as separated, small glyph-like star plots arranged in an array. The matrix view provides an overview, gives access to individual data objects, and supports users to reveal patterns or anomalies within the data. Both views are logically linked to each other (interplay). Interacting with one of them can cause changes in the other (e.g., object selection, rearrange axes). Consequently, we expect users to focus more on the detail view than the matrix view. As some devices provide limited display space (e.g., mobile phones, tablets), the matrix view can also be hidden (i.e., by dragging it to the screen border).

\section{Select \& Explore}

Many interface elements (e.g., text labels, axes, stars and their contours) can be selected by tapping on them. To inspect properties of individual objects, users select them by tapping on a star plot in the matrix view. Selected objects are highlighted in the matrix and shown in the detail view. An object can also be dragged from the matrix to the detail view. In detail view, objects (i.e., the stars) can be highlighted by tapping it or its contour.

Reading of exact attribute values in the detail view can be achieved in various ways. If a star is selected, labels
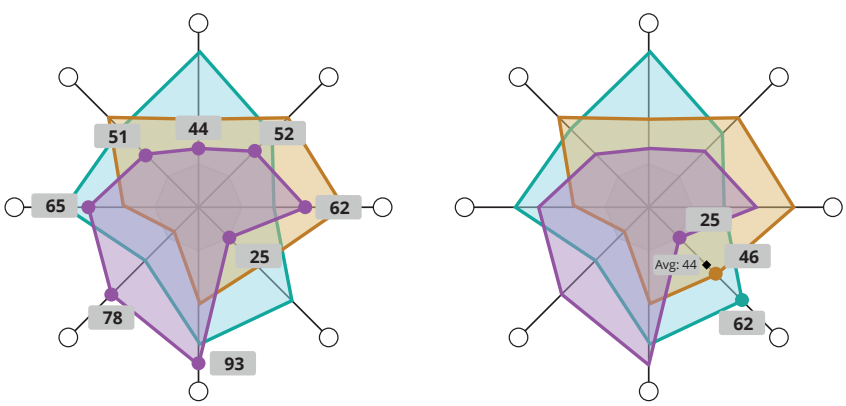

Figure 5: Attribute values of a single object (left), attribute values along a specific axis (right).

show all values of the selected star on shape-axes intersections (Figure 5, left). Tapping on an axis (selection) only shows values along the selected axis (Figure 5, right). In addition, an extra annotation on the selected axis informs about the mean value. Similar to slider and range visualization in DataMeadow [5], this could also be the distribution of objects. To explore objects and read individual values, users can touch and hold the background of the plot and start dragging. While moving the finger across the plot, a selection mark highlights the data point (object-axis intersection) closest to the position of the finger and shows the corresponding value. The selection mark automatically disappears when the finger is released.

\section{Reconfigure}

The matrix view allows users to rearrange objects. Users can manually define a specific position in the matrix by touching, holding, and then dragging a star plot. In addition, all objects can be sorted using a pre-defined attribute. If one of the axes in the detail view is selected, users can swipe downwards with two fingers to sort all data objects in a descending order. An upward swipe results in an ascending order. 


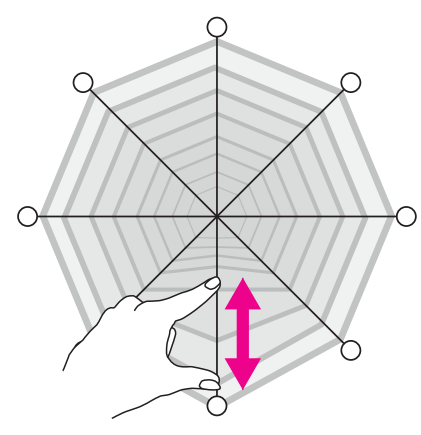

Figure 6: Fisheye distortion applied to an axis.

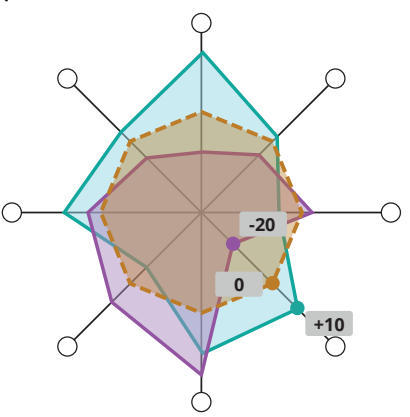

Figure 7: Relative objects presentation.

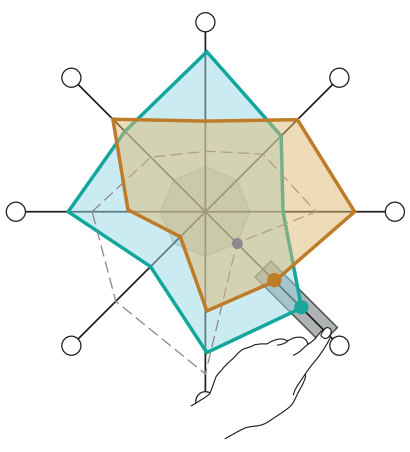

Figure 8: Filter area can be used to define a ranges.
Due to the nature of coordinate plots, the arrangement of axes is critical if users want to reveal, e.g., relations between attributes. Furthermore, the shape of a star depends on this order; and this might help to improve the identification of patterns or anomalies in the matrix view. To move a single axis, users touch and drag an axis (or a handle attached to each axis) in the detail view. Releasing the finger confirms the new position, resulting in a rearrangement and equal distribution of all axes. If two fingers are used (two finger drag), all axes are pushed in the direction of the movement (Figure 3). This creates a gap in the plot and pushes together axes. To prevent one of the axes to rearrange, its position can be locked by touching and holding it for a short time. The state of this lock mode is indicated by the handle of the axis (fill color). Wiping across the plot (Figure 4) restores the equal distribution of axes. All changes regarding the arrangement of axes in the detail view are synchronized with the matrix view. This synchronization, however, can also be deactivated by using an option button in the matrix view.

\section{Encode}

To address encoding tasks, we propose two concepts: changing the scaling of an axis and switching between absolute and relative presentation. The scaling of an axis (in the detail view) can be changed by performing a pinch gesture on it. This gesture applies a fisheye distortion to the axis (Figure 6) and allows users to zoom in and out.

Furthermore, the detail view can present objects relatively to a single selected object. Users can specify a reference object by double-tapping it. The reference object appears with a uniform shape; all remaining stars are displayed using the difference regarding the reference object (Figure 7). To switch back to an absolute presentation, users simply tap the reference object twice again (double-tap). The tran- sition between these states makes use of smooth animations and allows users to visually follow the re-encoding.

\section{Abstract/Elaborate}

In this part, we propose a new approach for presenting (semi-)structured data with star plots. Our concept allows users to explore data at different levels in a single star plot visualization. If users double-tap the handle of an axis in the detail view, the axis (level 1) splits up and new axes appear in between (e.g., Figure 9). These sub-axes (level 2) represent attributes from another information layer. For instance, if objects represent countries (shape) defined by the number of car accidents per year (attributes, level 1 axes), than level 2 axes could represent data of various causes of accidents for the corresponding year. This approach adds the extra data points to the data objects and therefore changes the shapes of visible stars.

\section{Filter \& Connect}

We propose different filter and connect interactions performed in the detail view. To modify the shape of objects users can remove axes from the plot by swiping the handle of an axis outwards (i.e., away from the center of the plot). Inspired by brushing from parallel coordinates, users can specify a range (or condition) on a specific axis in order to filter the objects presented in the matrix view. Touching and holding an axis with two fingers for a short time creates a rectangular filter area along the axis (Figure 8). This filter area is not restricted to objects shown in the detail view. It directly filters (e.g., fades out) objects outside of the range. The position of the area can be adjusted afterwards by dragging it along the axis. To change the expanse of the area users simply touch and drag the area's lower or upper border.

In contrast to the 'more permanent' filter area, users can touch and drag along an axis to temporally show objects 


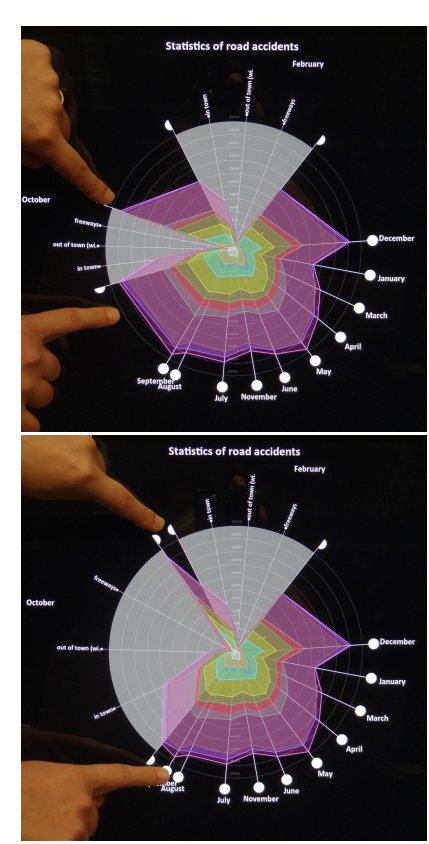

Figure 9: Increasing the area of sub-axes. that correspond to the specified attribute value. While the detail view shows a flimsy contour of corresponding objects the matrix clearly highlights them.

\section{Implementation}

Our prototype runs on a 27" WQHD display from Perceptive Pixel, supporting both pen and multi-touch input. It is driven by an ordinary desktop computer (i73.4 GHz) running Windows. User interface and visualization components are written in Python using the media-centric application framework libavg ${ }^{1}$. Our implementation visualizes four different data sets from Destatis ${ }^{2}$ (the Federal Statistical Office of Germany): students, family structures, car accidents, and marriages ${ }^{3}$.

At the moment of writing only parts of our concepts have been implemented. Focused on the detail view, our first prototype features many basic functionalities such as selecting one or multiple objects, showing and hiding specific attributes (axes), and showing specific value along axes. Furthermore, users can rearrange axes: drag a single axis as well as push multiple axes (Figure 10). Finally, we implemented our proposed concept of splitting axes (Figure 9) to further explore other levels of information.

\section{Conclusion}

We presented a systematic and consistent set of multitouch interactions for star plot visualizations. We focused on natural and fluid touch interactions addressing several visualization tasks. In addition, we propose a new approach to present and interact with (semi-)structured data within a star plot. Finally, we successfully implemented many of our concepts in a first prototype.

\section{${ }^{1} \mathrm{https}: / /$ www.libavg.de/ \\ ${ }^{2} \mathrm{https}: / /$ www.destatis.de/EN/}

${ }^{3}$ Data codes: 21311-0006, 12211-0602, 46241-0011, 12611-0005
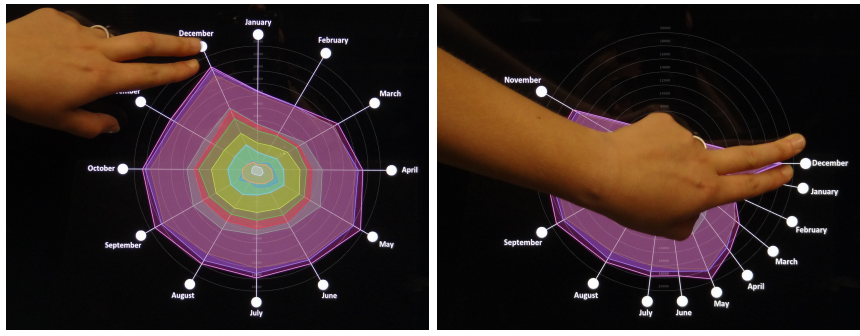

Figure 10: Moving multiple axes using a two-finger drag.

At the current point in time, there has been no formal testing. For future work, we plan to evaluate our concepts by comparing it to a traditional star plot interface and to further investigate new visual extensions for star plots. We want to implement additional concepts and enhance the prototype to better support touch-enabled devices of different size. While our concepts are specifically designed for star plots, we think some of the principles and interactions proposed in our work can be transferred to other visualization techniques.

\section{Acknowledgements}

We thank our lab students Tamara Flemisch and Philip Manja for their conceptional as well as programming assistance. We also thank our colleague Wolfgang Büschel for his continuous support. This work has partially been funded by the DFG through the project GEMS (DA 1319/3-1).

\section{REFERENCES}

1. Dominikus Baur, Bongshin Lee, and Sheelagh Carpendale. 2012. TouchWave: Kinetic Multi-touch Manipulation for Hierarchical Stacked Graphs. In Proc. ITS 2012. ACM, 255-264.

2. William Buxton. 1986. Chunking and Phrasing and the Design of Human-Computer Dialogues. In Proc. IFIP 
World Computer Congress. 475-480.

3. Steven M. Drucker, Danyel Fisher, Ramik Sadana, Jessica Herron, and m.c. schraefel. 2013. TouchViz: A Case Study Comparing Two Interfaces for Data Analytics on Tablets. In Proc. CHI 2013. ACM, 2301-2310.

4. Niklas Elmqvist, Andrew Vande Moere, Hans-Christian Jetter, Daniel Cernea, Harald Reiterer, and T.-J. Jankun-Kelly. 2011. Fluid Interaction for Information Visualization. Information Visualization 10, 4 (2011), 327-340.

5. Niklas Elmqvist, John Stasko, and Philippas Tsigas. 2008. DataMeadow: A Visual Canvas for Analysis of Large-Scale Multivariate Data. Information Visualization 7, 1 (2008), 18-33.

6. Bongshin Lee, Petra Isenberg, Nathalie Henry Riche, and Sheelagh Carpendale. 2012. Beyond Mouse and Keyboard: Expanding Design Considerations for Information Visualization Interactions. Visualization and
Computer Graphics, IEEE Transactions on 18, 12 (2012), 2689-2698.

7. Jeffrey M. Rzeszotarski and Aniket Kittur. 2014. Kinetica: Naturalistic Multi-touch Data Visualization. In Proc. CHI 2014. ACM, 897-906.

8. Ramik Sadana and John Stasko. 2014. Designing and Implementing an Interactive Scatterplot Visualization for a Tablet Computer. In Proc. AVI 2014. ACM, 265-272.

9. Matthew O. Ward. 2008. Multivariate Data Glyphs: Principles and Practice. In Handbook of Data Visualization. Springer Berlin Heidelberg, 179-198.

10. Ji Soo Yi, Youn ah Kang, J.T. Stasko, and J.A. Jacko. 2007. Toward a Deeper Understanding of the Role of Interaction in Information Visualization. Visualization and Computer Graphics, IEEE Transactions on 13, 6 (Nov 2007), 1224-1231. 